\title{
Suzuki's type fixed point theorems for generalized mappings in partial cone metric spaces over a solid cone
}

\author{
Wenqing $\mathrm{Xu}^{1,2}$, Chuanxi Zhu ${ }^{1 *}$ and Chunfang Chen ${ }^{1}$
}

\section{"Correspondence:}

chuanxizhu@126.com

'Department of Mathematics,

Nanchang University, Nanchang,

330031, P.R. China

Full list of author information is

available at the end of the article

\section{每 Springer}

\begin{abstract}
In this paper, we obtain some Suzuki-type fixed point theorems for generalized mappings in partial cone metric spaces over a solid cone. Our results unify and generalize various known comparable results in the literature. We also provide illustrative examples in support of our new results.
\end{abstract}

MSC: $47 \mathrm{H} 10 ; 54 \mathrm{H} 25$

Keywords: partial cone metric spaces; solid cone; Suzuki type; fixed point

\section{Introduction and preliminaries}

In 1994, Matthews [1] introduced the notion of a partial metric space as a part of the study of denotational semantics of data for networks, showing that the Banach contraction mapping principle can be generalized to the partial metric context for applications in program verification. After that, many fixed point theorems for mappings satisfying different contractive conditions in (ordered) partial metric spaces have been proved (see [2-4]).

In 2007, Huang and Zhang [5] introduced the concept of cone metric spaces and extended the Banach contraction principle to cone spaces over a normal solid cone. Moreover, they defined the convergence via interior points of the cone. Such an approach allows the investigation of the case that the cone is not necessarily normal. Since then, there were many references concerned with fixed point results in (ordered) cone spaces (see [6-15]). In 2012, based on the definition of cone metric spaces and partial metric spaces, Sonmez $[16,17]$ defined a partial cone metric space and proved some fixed point theorems for contractive type mappings in complete partial cone metric spaces.

Recently, without using the normality of the cone, Malhotra et al. [18] and Jiang and Li $[19]$ extended the results of $[16,17]$ to $\theta$-complete partial cone metric spaces.

First, we recall the definition of partial metric spaces (see [1]).

Definition 1.1 ([1]) Let $X$ be a nonempty set. A function $p: X \times X \rightarrow \mathbb{R}^{+}$is said to be a partial metric if for all $x, y, z \in X$, the following conditions are satisfied:

(p1) $p(x, x)=p(x, y)=p(y, y)$ if and only if $x=y$;

(p2) $p(x, x) \leq p(x, y)$

(p3) $p(x, y)=p(y, x)$;

(c) $2016 \mathrm{Xu}$ et al. This article is distributed under the terms of the Creative Commons Attribution 4.0 International License (http://creativecommons.org/licenses/by/4.0/), which permits unrestricted use, distribution, and reproduction in any medium, provided you give appropriate credit to the original author(s) and the source, provide a link to the Creative Commons license, and indicate if changes were made. 
(p4) $p(x, y) \leq p(x, z)+p(z, y)-p(z, z)$.

The pair $(X, p)$ is called a partial metric space. If $p(x, y)=0$, then the $(p 1)$ and $(p 2)$ imply that $x=y$, but the converse does not hold in general. A trivial example of a partial metric space is the pair $\left(\mathbb{R}^{+}, p\right)$, where $p: \mathbb{R}^{+} \times \mathbb{R}^{+} \rightarrow \mathbb{R}^{+}$is defined as $p(x, y)=\max \{x, y\}$; see also [1].

Let $E$ be a topological vector space. A cone of $E$ is a nonempty closed subset $P$ of $E$ such that

(i) $a x+b y \in P$ for all $x, y \in P$ and $a, b \geq 0$, and

(ii) $P \cap(-P)=\{\theta\}$, where $\theta$ is the zero element of $E$.

Each cone $P$ of $E$ determines a partial order $\preceq$ on $E$ by $x \preceq y$ if and only if $y-x \in P$ for all $x, y \in E$. We shall write $x \prec y$ if $x \preceq y$ and $x \neq y$.

A cone $P$ of a topological vector space $E$ is solid if int $P \neq \varnothing$, where $\operatorname{int} P$ is the interior of $P$. For all $x, y \in E$ with $y-x \in \operatorname{int} P$, we write $x \ll y$. Let $P$ be a solid cone of a topological vector space $E$. A sequence $\left\{u_{n}\right\}$ of $E$ weakly converges [18] to $u \in E$ (denoted $u_{n} \stackrel{w}{\rightarrow} u$ ) if for each $c \in \operatorname{int} P$, there exists a positive integer $n_{0}$ such that $u-c \ll u_{n} \ll u+c$ for all $n \geq n_{0}$. A cone $P$ of a normed vector space $(E,\|\cdot\|)$ is normal if there exists $K>0$ such that $\theta \preceq x \preceq y$ implies that $\|x\| \leq K\|y\|$ for all $x, y \in P$, and the minimal $K$ is called a normal constant of $P$. Next, we state the definitions of cone metric and partial cone metric spaces and some of their properties (see [5, 16-19]).

Definition 1.2 ([5]) Let $X$ be a nonempty set, and let $P$ be a cone of a topological vector space $E$. A cone metric on $X$ is a mapping $d: X \times X \rightarrow P$ such that, for all $x, y, z \in X$ :

(d1) $d(x, y)=\theta$ if and only if $x=y$;

(d2) $d(x, y)=d(y, x)$;

(d3) $d(x, y) \preceq d(x, z)+d(z, y)$.

The pair $(X, d)$ is called a cone metric space over $P$.

Definition $1.3([16,17])$ Let $X$ be a nonempty set, and let $P$ be a cone of a topological vector space $E$. A partial cone metric on $X$ is a mapping $p: X \times X \rightarrow P$ such that, for all $x, y, z \in X$ :

(p1) $p(x, x)=p(x, y)=p(y, y)$ if and only if $x=y$;

(p2) $p(x, x) \preceq p(x, y)$;

(p3) $p(x, y)=p(y, x)$;

(p4) $p(x, y) \preceq p(x, z)+p(z, y)-p(z, z)$.

In this case, the pair $(X, p)$ is called a partial cone metric space over $P$.

Note that each cone metric is certainly a partial cone metric. The following example shows that there do exist partial cone metrics that are not cone metrics.

Example 1.1 ([19]) Let $E=C_{R}^{1}[0,1]$ with the norm $\|u\|=\|u\|_{\infty}+\left\|u^{\prime}\right\|_{\infty}$, and $X=P=\{u \in$ $E: u(t) \geq 0, t \in[0,1]\}$, which is a nonnormal solid cone. Define the mapping $p: X \times X \rightarrow P$ by 


$$
p(x, y)= \begin{cases}x, & x=y \\ x+y & \text { otherwise }\end{cases}
$$

Then $p$ is a partial cone metric, but not a cone metric, since $p(x, x) \neq \theta$ for all $x \in X$ with $x \neq \theta$.

A partial cone metric $p$ on $X$ over a solid cone $P$ generates a topology $\tau_{p}$ on $X$, which has a base of the family of open $p$-balls $\left\{B_{p}(x, c): x \in X, \theta \ll c\right\}$, where $B_{p}(x, c)=\{y \in X$ : $p(x, y) \ll p(x, x)+c\}$ for $x \in X$ and $c \in \operatorname{int} P$.

Definition 1.4 ([19]) Let $(X, p)$ be a partial cone metric space over a solid cone $P$ of a topological vector space $E$.

(i) A sequence $\left\{x_{n}\right\}$ in $X$ converges to $x \in X$ (denoted by $x_{n} \stackrel{\tau_{p}}{\rightarrow} x$ ) if for each $c \in \operatorname{int} P$, there exists a positive integer $n_{0}$ such that $p\left(x_{n}, x\right) \ll p(x, x)+c$ for each $n \geq n_{0}$ (that is, $\left.p\left(x_{n}, x\right) \stackrel{w}{\rightarrow} p(x, x)\right)$.

(ii) A sequence $\left\{x_{n}\right\}$ in $X$ is $\theta$-Cauchy if for each $c \in \operatorname{int} P$, there exists a positive integer $n_{0}$ such that $p\left(x_{n}, x_{m}\right) \ll c$ for all $m, n \geq n_{0}$. The partial cone metric space $(X, p)$ is $\theta$-complete if each $\theta$-Cauchy sequence $\left\{x_{n}\right\}$ of $X$ converges to a point $x \in X$ such that $p(x, x)=\theta$.

Definition $1.5([16,17])$ Let $(X, p)$ be a partial cone metric space over a solid cone $P$ of a topological vector space $(E,\|\cdot\|)$.

(i) A sequence $\left\{x_{n}\right\}$ in $X$ strongly converges to $x \in X$ (denoted by $x_{n} \stackrel{s-\tau_{p}}{\rightarrow} x$ ) if

$$
\lim _{n \rightarrow \infty} p\left(x_{n}, x\right)=\lim _{n \rightarrow \infty} p\left(x_{n}, x_{n}\right)=p(x, x)
$$

(ii) A sequence $\left\{x_{n}\right\}$ in $X$ is Cauchy if there exists $u \in P$ with $\|u\|<\infty$ such that $\lim _{m, n \rightarrow \infty} p\left(x_{m}, x_{n}\right)=u$. The partial cone metric space $(X, p)$ is complete if each Cauchy sequence $\left\{x_{n}\right\}$ of $X$ strongly converges to a point $x \in X$ such that $p(x, x)=u$.

Note that if $P$ is a normal solid cone of a normed vector space $(E,\|\cdot\|)$, then each complete partial cone metric space is $\theta$-complete. But the converse is not true. The following example ([16], Example 4) shows that a $\theta$-complete partial cone metric space is not necessarily complete.

Example 1.2 ([16]) Let $X=\left\{\left(x_{1}, x_{2}, \ldots, x_{k}\right): x_{i} \geq 0, x_{i} \in Q, i=1,2, \ldots, k\right\}$, and $E=R^{k}$ with the norm $\|x\|=\sqrt{\sum_{i=1}^{k} x_{i}^{2}}, P=R_{+}^{k}$, where $Q$ denotes the set of rational numbers. Define the mapping $p: X \times X \rightarrow P$ by

$$
p(x, y)=\left(x_{1} \vee y_{1}, x_{2} \vee y_{2}, \ldots, x_{k} \vee y_{k}\right) \quad \text { for all } x, y \in X \text {, }
$$

where the symbol $\vee$ denotes the maximum, that is, $x \vee y=\max \{x, y\}$. Clearly, $(X, p)$ is a partial cone metric space, $p(x, x)=x$ for each $x \in X, p(x, \theta)=\theta$ if and only if $x=\theta$, and $P$ is normal. On the other hand, $(X, p)$ is $\theta$-complete but not complete.

Let $X$ be a nonempty set, and $S, T: X \rightarrow X$ be two mappings. A point $x \in X$ is said to be a coincidence point of $S$ and $T$ if $S x=T x$. A point $y \in X$ is called point of coincidence 
of $S$ and $T$ if there exists a point $x \in X$ such that $y=S x=T x$. The mappings $S, T$ are said to be weakly compatible if they commute at their coincidence points (that is, $T S z=S T z$ whenever $S z=T z)$.

Let $(X, \sqsubseteq)$ be a partially ordered set; $x, y \in X$ are called comparable if $x \sqsubseteq y$ or $y \sqsubseteq x$. A mapping $T: X \rightarrow X$ is said to be nondecreasing if for $x, y \in X, x \sqsubseteq y$ implies $T x \sqsubseteq T y$. Let $S, T: X \rightarrow X$ be two mappings; $T$ is said to be $S$-nondecreasing if $S x \sqsubseteq S y$ implies $T x \sqsubseteq T y$ for all $x, y \in X$.

Bhasker and Lakshmikantham [20] introduced the concepts of mixed monotone mappings and coupled fixed point.

Definition 1.6 ([20]) Let $(X$, ᄃ) be a partially ordered set, and $A: X \times X \rightarrow X$. The mapping $A$ is said to have the mixed monotone property if $A$ is monotone nondecreasing in its first argument and is monotone nonincreasing in its second argument, that is, for any $x, y \in X$,

$$
\begin{array}{ll}
x_{1}, x_{2} \in X, & x_{1} \sqsubseteq x_{2} \quad \Longrightarrow \quad A\left(x_{1}, y\right) \sqsubseteq A\left(x_{2}, y\right), \\
y_{1}, y_{2} \in X, & y_{2} \sqsubseteq y_{1} \quad \Longrightarrow \quad A\left(x, y_{1}\right) \sqsubseteq A\left(x, y_{2}\right) .
\end{array}
$$

Definition 1.7 ([20]) An element $(x, y) \in X^{2}$ is said to be a coupled fixed point of the mapping $A: X^{2} \rightarrow X$ if $A(x, y)=x$ and $A(y, x)=y$.

Lemma 1.1 ([21]) Let $X$ be a nonempty set, and $S: X \rightarrow X$ a mapping. Then there exists a subset $Y \subseteq X$ such that $S Y=S X$ and $S: Y \rightarrow X$ is one-to-one.

Paesano and Vetro [22] proved Suzuki-type characterizations of completeness for partial metric spaces and fixed points for partially ordered metric spaces. Note that if in Theorem 2 of [22], we assume that $p$ is a metric, then we obtain Theorem 2 of [23]. Recently, also, some Suzuki-type fixed point and coupled fixed point results for mappings or generalized multivalued mappings in different metric spaces were investigated (see [24-29]). The aim of the paper is to give a generalized version of Theorems 2 and 3 of [22] in partially ordered partial cone metric spaces over a solid cone. Meantime, we also establish the corresponding Suzuki-type coupled fixed point results for generalized mappings in partially ordered partial cone metric spaces. It is worth pointing out that some examples are presented to verify the effectiveness and applicability of our results.

\section{Fixed point theorems in partial cone metric spaces}

In this section, we first give some properties of partial cone metric spaces. The following properties are used (particularly when dealing with cone metric spaces in which the cone need not be normal).

Remark 2.1 Let $P$ be a solid cone. Then the following properties are used:

(1) If $a \preceq b$ and $b \preceq c$, then $a \preceq c$.

(2) If $a \ll b$ and $b \ll c$, then $a \ll c$.

(3) If $\theta \preceq u \ll c$ for each $c \in \operatorname{int} P$, then $u=\theta$.

(4) If $a \preceq \lambda a$, where $0 \leq \lambda<1$, then $a=\theta$.

(5) If $a \preceq b+c$ for each $c \in \operatorname{int} P$, then $a \preceq b$. 
Now, we establish some Suzuki-type fixed point theorems for generalized mappings in partially ordered partial cone metric spaces over a solid cone.

Theorem 2.1 Let $(X, p$, $\sqsubseteq)$ be a $\theta$-complete partially ordered partial cone metric space over a solid cone $P$ of a normed vector space $(E,\|\cdot\|)$. Let $T: X \rightarrow X$ be a nondecreasing mapping with respect to $\sqsubseteq$. Define the nonincreasing function $\psi:[0,1) \rightarrow\left(\frac{1}{2}, 1\right]$ by

$$
\psi(r)= \begin{cases}1 & \text { if } 0 \leq r<\frac{\sqrt{5}-1}{2} \\ \frac{1-r}{r^{2}} & \text { if } \frac{\sqrt{5}-1}{2} \leq r<\frac{\sqrt{2}}{2}, \\ \frac{1}{1+r} & \text { if } \frac{\sqrt{2}}{2} \leq r<1\end{cases}
$$

Assume that there exists $r \in[0,1)$ such that

$$
\psi(r) p(x, T x) \preceq p(x, y) \quad \text { implies } \quad p(T x, T y) \preceq r U(x, y)
$$

for all comparable $x, y \in X$, where $U(x, y) \in\left\{p(x, y), p(x, T x), p(y, T y), \frac{p(x, T y)+p(y, T x)}{2}\right\}$. Suppose that the following conditions hold:

(i) there exists $x_{0} \in X$ such that $x_{0} \sqsubseteq T x_{0}$;

(ii) for a nondecreasing sequence $x_{n} \stackrel{\tau_{p}}{\rightarrow} x$, we have $x_{n} \sqsubseteq x$ for all $n \in N$;

(iii) for two nondecreasing sequences $\left\{x_{n}\right\},\left\{y_{n}\right\} \subseteq X$ such that $x_{n} \subseteq y_{n}, x_{n} \stackrel{\tau_{p}}{\rightarrow} x$, and $y_{n} \stackrel{\tau_{p}}{\rightarrow} y$ as $n \rightarrow \infty$, we have $x \sqsubseteq y$.

Then $T$ has a fixed point in X. Moreover, the fixed point of $T$ is unique if

(iv) for all $x, y \in X$ that are not comparable, there exists $u \in X$ comparable with $x$ and $y$.

Proof Since $\psi(r) \leq 1, \psi(r) p(x, T x) \preceq p(x, T x)$ for every $x \in X$. By (2.1) and using the triangular inequality, we have

$$
p\left(T x, T^{2} x\right) \preceq r U(x, T x),
$$

where

$$
\begin{aligned}
U(x, T x) & \in\left\{p(x, T x), p(x, T x), p\left(T x, T^{2} x\right), \frac{p\left(x, T^{2} x\right)+p(T x, T x)}{2}\right\} \\
& =\left\{p(x, T x), p\left(T x, T^{2} x\right), \frac{p\left(x, T^{2} x\right)+p(T x, T x)}{2}\right\} .
\end{aligned}
$$

Thus, we get the following cases:

Case 1. $p\left(T x, T^{2} x\right) \preceq r p(x, T x)$.

Case 2. $p\left(T x, T^{2} x\right) \preceq r p\left(T x, T^{2} x\right)$, which implies that $p\left(T x, T^{2} x\right)=\theta$.

Case 3. $p\left(T x, T^{2} x\right) \preceq r \frac{p\left(x, T^{2} x\right)+p(T x, T x)}{2} \preceq r \frac{p(x, T x)+p\left(T x, T^{2} x\right)}{2}$, which implies that $p\left(T x, T^{2} x\right) \preceq r p(x, T x)$.

Then, in all cases, we have

$$
p\left(T x, T^{2} x\right) \preceq r p(x, T x) .
$$

Let $x_{0} \in X$ be such that $x_{0} \sqsubseteq T x_{0}$. Since $T$ is nondecreasing, we get

$$
x_{0} \sqsubseteq T x_{0} \sqsubseteq T^{2} x_{0} \sqsubseteq \cdots \sqsubseteq T^{n} x_{0} \sqsubseteq \cdots .
$$


Define the sequence $\left\{x_{n}\right\}$ by $x_{n}=T^{n} x_{0}$, so that $x_{n+1}=T x_{n}$. If $x_{n}=x_{n+1}=T x_{n}$ for some $n$, then $x_{n}$ becomes a fixed point of $T$. Now, suppose that $x_{n} \neq x_{n+1}$ for all $n \in N$. Then $p\left(x_{n}, x_{n+1}\right) \succ \theta$.

Note that $\psi(r) p\left(x_{n-1}, T x_{n-1}\right) \preceq p\left(x_{n-1}, T x_{n-1}\right)$ for all $n \in Z^{+}$, where $Z^{+}$is the set of positive integers. Since $x_{n-1}$ and $T x_{n-1}$ are comparable for all $n>0$, by (2.1) we have

$$
p\left(x_{n}, x_{n+1}\right)=p\left(T x_{n-1}, T^{2} x_{n-1}\right) \preceq r U\left(x_{n-1}, T x_{n-1}\right)
$$

where

$$
\begin{aligned}
U\left(x_{n-1}, T x_{n-1}\right) \in & \left\{p\left(x_{n-1}, T x_{n-1}\right), p\left(x_{n-1}, T x_{n-1}\right), p\left(T x_{n-1}, T^{2} x_{n-1}\right),\right. \\
& \left.\frac{p\left(x_{n-1}, T^{2} x_{n-1}\right)+p\left(T x_{n-1}, T x_{n-1}\right)}{2}\right\} \\
= & \left\{p\left(x_{n-1}, x_{n}\right), p\left(x_{n}, x_{n+1}\right), \frac{p\left(x_{n-1}, x_{n+1}\right)+p\left(x_{n}, x_{n}\right)}{2}\right\} .
\end{aligned}
$$

Thus, we get the following cases:

Case 1. If $U\left(x_{n-1}, T x_{n-1}\right)=p\left(x_{n-1}, x_{n}\right)$, then $p\left(x_{n}, x_{n+1}\right) \preceq r p\left(x_{n-1}, x_{n}\right)$.

Case 2. $p\left(x_{n}, x_{n+1}\right) \preceq r p\left(x_{n}, x_{n+1}\right)$, which implies that $p\left(x_{n}, x_{n+1}\right)=\theta$.

Case 3. $p\left(x_{n}, x_{n+1}\right) \preceq r \cdot \frac{p\left(x_{n-1}, x_{n+1}\right)+p\left(x_{n}, x_{n}\right)}{2} \preceq r\left(\frac{p\left(x_{n-1}, x_{n}\right)+p\left(x_{n}, x_{n+1}\right)}{2}\right)$, which implies that $p\left(x_{n}, x_{n+1}\right) \preceq r p\left(x_{n-1}, x_{n}\right)$.

Then, in all cases, we have

$$
p\left(x_{n}, x_{n+1}\right) \preceq r p\left(x_{n-1}, x_{n}\right) .
$$

Continuing this process, it follows that

$$
p\left(x_{n}, x_{n+1}\right) \preceq r p\left(x_{n-1}, x_{n}\right) \preceq r^{2} p\left(x_{n-2}, x_{n-1}\right) \preceq \cdots \preceq r^{n} p\left(x_{0}, x_{1}\right)
$$

Thus, for any $m, n \in Z^{+}$with $m>n$, we get

$$
\begin{aligned}
p\left(x_{n}, x_{m}\right) & \preceq p\left(x_{n}, x_{n+1}\right)+p\left(x_{n+1}, x_{n+2}\right)+\cdots+p\left(x_{m-1}, x_{m}\right) \\
& \preceq\left(r^{n}+r^{n+1}+\cdots+r^{m-1}\right) p\left(x_{0}, x_{1}\right) \\
& \preceq \frac{r^{n}}{1-r} p\left(x_{0}, x_{1}\right) .
\end{aligned}
$$

Let $\theta \ll c$ be given, Choose $\delta>0$ such that $c+N_{\delta}(\theta) \subseteq P$, where $N_{\delta}(\theta)=\{y \in E$ : $\|y\|<\delta\}$. Also, choose a natural number $N_{1}$ such that $\frac{r^{n}}{1-r} p\left(x_{0}, x_{1}\right) \in N_{\delta}(\theta)$ for all $n \geq N_{1}$. Then $\frac{r^{n}}{1-r} p\left(x_{0}, x_{1}\right) \ll c$ for all $n \geq N_{1}$. Thus,

$$
p\left(x_{n}, x_{m}\right) \preceq \frac{r^{n}}{1-r} p\left(x_{0}, x_{1}\right) \ll c
$$

for all $m>n \geq N_{1}$. Hence, $\left\{x_{n}\right\}$ is a $\theta$-Cauchy sequence in $(X, p)$. Since $(X, p)$ is a $\theta$-complete partial cone metric space, there exists $z \in X$ such that $x_{n} \stackrel{\tau_{p}}{\rightarrow} z$ and $p(z, z)=\theta$.

First, we show that there exists $j \in Z^{+}$such that $T^{j} z=z$. Arguing by contradiction, we assume that $T^{j} z \neq z$ for all $j \in Z^{+}$. 
Note that, by condition (ii), if $\left\{x_{n}\right\}$ is nondecreasing, then $x_{n} \sqsubseteq z$. Since $T$ is nondecreasing, we get $x_{n+1}=T x_{n} \sqsubseteq T z$ for all $n \in N$. Taking the limit as $n \rightarrow \infty$, by (iii) we obtain that $z \sqsubseteq T z$, which implies that $\left\{T^{n} z\right\}$ is a nondecreasing sequence. So, we have shown that for $\left\{x_{n}\right\},\left\{T^{n} z\right\}$ also is a $\theta$-Cauchy sequence. We also have $T^{j} z$ is comparable with $x_{n}$ for all $j, n \in N$.

Now, we prove that

$$
p\left(T^{j+1} z, z\right) \preceq r^{j} p(T z, z) \quad \text { for all } j \in N \text {. }
$$

Since $p\left(T^{j} z, z\right) \succ \theta, p(z, z)=\theta$, and $x_{n} \stackrel{\tau_{p}}{\rightarrow} z$, there exists $N_{2} \in N$ such that $p\left(x_{n}, z\right) \preceq \frac{p\left(T^{j} z, z\right)}{3}$ for all $n \geq N_{2}$. We have

$$
\begin{aligned}
\psi(r) p\left(x_{n}, T x_{n}\right) & \preceq p\left(x_{n}, T x_{n}\right) \preceq p\left(x_{n}, z\right)+p\left(x_{n+1}, z\right) \\
& \preceq \frac{2}{3} p\left(T^{j} z, z\right)=p\left(T^{j} z, z\right)-\frac{1}{3} p\left(T^{j} z, z\right) \\
& \preceq p\left(T^{j} z, z\right)-p\left(x_{n}, z\right) \preceq p\left(x_{n}, T^{j} z\right) .
\end{aligned}
$$

By (2.1) and using the triangle inequality, we get that

$$
p\left(x_{n+1}, T^{j+1} z\right) \preceq r U\left(x_{n}, T^{j} z\right),
$$

where

$$
U\left(x_{n}, T^{j} z\right) \in\left\{p\left(x_{n}, T^{j} z\right), p\left(x_{n}, x_{n+1}\right), p\left(T^{j} z, T^{j+1} z\right), \frac{p\left(x_{n}, T^{j+1} z\right)+p\left(T^{j} z, x_{n+1}\right)}{2}\right\} .
$$

Thus,

$$
\begin{aligned}
p\left(z, T^{j+1} z\right) & \preceq p\left(x_{n+1}, z\right)+p\left(x_{n+1}, T^{j+1} z\right) \\
& \preceq p\left(x_{n+1}, z\right)+r U\left(x_{n}, T^{j} z\right),
\end{aligned}
$$

where

$$
U\left(x_{n}, T^{j} z\right) \in\left\{p\left(x_{n}, T^{j} z\right), p\left(x_{n}, x_{n+1}\right), p\left(T^{j} z, T^{j+1} z\right), \frac{p\left(x_{n}, T^{j+1} z\right)+p\left(T^{j} z, x_{n+1}\right)}{2}\right\} .
$$

Since $x_{n} \stackrel{\tau_{p}}{\rightarrow} z$ for every $c \gg \theta$, there exists $n_{0} \in N$ such that $p\left(x_{n}, z\right) \ll \frac{c}{2}$ and $p\left(x_{n}, x_{n+1}\right) \ll \frac{c}{2}$ for all $n>n_{0}$. Now, for $n>n_{0}$, we consider the following cases:

Case 1. $p\left(z, T^{j+1} z\right) \preceq p\left(x_{n+1}, z\right)+r p\left(x_{n}, T^{j} z\right) \preceq p\left(x_{n+1}, z\right)+r\left(p\left(x_{n}, z\right)+p\left(z, T^{j} z\right)\right) \preceq r p(z$, $\left.T^{j} z\right)+c$. Then it follows from Remark 2.1(5) that $p\left(z, T^{j+1} z\right) \preceq r p\left(z, T^{j} z\right)$, and we get

$$
p\left(z, T^{j+1} z\right) \preceq r p\left(z, T^{j} z\right) \preceq r^{2} p\left(z, T^{j-1} z\right) \preceq \cdots \preceq r^{j} p(z, T z) .
$$

Case 2. $p\left(z, T^{j+1} z\right) \preceq p\left(x_{n+1}, z\right)+r p\left(x_{n}, x_{n+1}\right) \ll c$, which implies that $p\left(z, T^{j+1} z\right)=\theta$.

Case 3.

$$
p\left(z, T^{j+1} z\right) \preceq p\left(x_{n+1}, z\right)+r p\left(T^{j} z, T^{j+1} z\right) \preceq c+r p\left(T^{j} z, T^{j+1} z\right)
$$

which implies that $p\left(z, T^{j+1} z\right) \preceq r p\left(T^{j} z, T^{j+1} z\right)$. Then from (2.2) we have 


$$
p\left(z, T^{j+1} z\right) \preceq r p\left(T^{j} z, T^{j+1} z\right) \preceq r^{2} p\left(T^{j-1} z, T^{j} z\right) \preceq \cdots \preceq r^{j+1} p(z, T z) \preceq r^{j} p(z, T z) .
$$

Case 4.

$$
\begin{aligned}
p\left(z, T^{j+1} z\right) & \preceq p\left(x_{n+1}, z\right)+r \cdot \frac{p\left(x_{n}, T^{j+1} z\right)+p\left(T^{j} z, x_{n+1}\right)}{2} \\
& \preceq p\left(x_{n+1}, z\right)+r \frac{p\left(x_{n}, z\right)+p\left(z, T^{j+1} z\right)+p\left(T^{j} z, z\right)+p\left(z, x_{n+1}\right)}{2} \\
& \ll c+r \cdot \frac{p\left(z, T^{j+1} z\right)+p\left(T^{j} z, z\right)}{2},
\end{aligned}
$$

which implies that $p\left(z, T^{j+1} z\right) \preceq r p\left(z, T^{j} z\right)$. Then from (2.4) we get $p\left(z, T^{j+1} z\right) \preceq r^{j} p(z, T z)$.

Thus, in all cases, we obtain $p\left(z, T^{j+1} z\right) \preceq r^{j} p(z, T z)$, that is, (2.3) holds.

Now, we consider the following three cases:

(1) $0 \leq r<\frac{\sqrt{5}-1}{2}$;

(2) $\frac{\sqrt{5}-1}{2} \leq r<\frac{\sqrt{2}}{2}$;

(3) $\frac{\sqrt{2}}{2} \leq r<1$.

In case (1), we note that $r^{2}+r<1$ and $\psi(r)=1$. If we assume that $p\left(T^{2} z, z\right) \prec p\left(T^{2} z, T^{3} z\right)$, then by (2.2) we have

$$
\begin{aligned}
p(z, T z) & \preceq p\left(z, T^{2} z\right)+p\left(T z, T^{2} z\right) \\
& \prec p\left(T^{2} z, T^{3} z\right)+p\left(T z, T^{2} z\right) \\
& \preceq r^{2} p(z, T z)+r p(z, T z) \\
& \preceq p(z, T z) .
\end{aligned}
$$

This is a contradiction. So, we have $p\left(T^{2} z, z\right) \succeq p\left(T^{2} z, T^{3} z\right)=\psi(r) p\left(T^{2} z, T^{3} z\right)$. By (2.1)(2.3) we deduce that

$$
p\left(T^{3} z, T z\right) \preceq r U\left(T^{2} z, z\right),
$$

where

$$
U\left(T^{2} z, z\right) \in\left\{p\left(T^{2} z, z\right), p\left(T^{2} z, T^{3} z\right), p(z, T z), \frac{p\left(T^{3} z, z\right)+p\left(T z, T^{2} z\right)}{2}\right\} .
$$

Thus, we get the following cases:

Case 1. $p\left(T^{3} z, T z\right) \preceq r p\left(T^{2} z, z\right) \preceq r^{2} p(T z, z) \preceq r p(T z, z)$.

Case 2. $p\left(T^{3} z, T z\right) \preceq r p\left(T^{2} z, T^{3} z\right) \preceq r^{3} p(T z, z) \preceq r p(T z, z)$.

Case 3. $p\left(T^{3} z, T z\right) \preceq r p(T z, z)$.

Case 4. $p\left(T^{3} z, T z\right) \preceq r \frac{p\left(T^{3} z, z\right)+p\left(T z, T^{2} z\right)}{2} \preceq r\left[\frac{r^{2} p(T z, z)+r p(z, T z)}{2}\right] \preceq r^{2} p(T z, z) \preceq r p(T z, z)$.

Then, in all cases, we have $p\left(T^{3} z, T z\right) \preceq r p(T z, z)$. Hence,

$$
\begin{aligned}
p(z, T z) & \preceq p\left(z, T^{3} z\right)+p\left(T^{3} z, T z\right) \\
& \preceq r^{2} p(z, T z)+r p(z, T z)=\left(r^{2}+1\right) p(z, T z) \\
& \prec p(z, T z),
\end{aligned}
$$

which is a contradiction. 
In case (2), we note that $2 r^{2}<1$ and $\psi(r)=\frac{1-r}{r^{2}}$. Now, we show by induction that

$$
p\left(T^{n} z, z\right) \preceq r p(z, T z)
$$

for $n \geq 2$. By (2.2), (2.5) holds for $n=2$. Assume that (2.5) holds for some $n$ with $n \geq 2$. Since

$$
p(z, T z) \preceq p\left(z, T^{n} z\right)+p\left(T^{n} z, T z\right) \preceq p\left(z, T^{n} z\right)+\operatorname{rp}(z, T z),
$$

we have

$$
p(z, T z) \preceq \frac{1}{1-r} p\left(z, T^{n} z\right),
$$

and so

$$
\begin{aligned}
\psi(r) p\left(T^{n} z, T^{n+1} z\right) & =\frac{1-r}{r^{2}} p\left(T^{n} z, T^{n+1} z\right) \preceq \frac{1-r}{r^{n}} p\left(T^{n} z, T^{n+1} z\right) \\
& \preceq(1-r) p(z, T z) \preceq p\left(z, T^{n} z\right) .
\end{aligned}
$$

Therefore, by the hypotheses we have

$$
p\left(T^{n+1} z, T z\right) \preceq r U\left(T^{n} z, T z\right),
$$

where

$$
U\left(T^{n} z, z\right) \in\left\{p\left(T^{n} z, z\right), p\left(T^{n} z, T^{n+1} z\right), p(z, T z), \frac{p\left(T^{n+1} z, z\right)+p\left(T z, T^{n} z\right)}{2}\right\}
$$

Thus, we get the following cases:

Case 1. $p\left(T^{n+1} z, T z\right) \preceq r p\left(T^{n} z, z\right) \preceq r^{n} p(T z, z) \preceq r p(T z, z)$.

Case 2. $p\left(T^{n+1} z, T z\right) \preceq r p\left(T^{n} z, T^{n+1} z\right) \preceq r^{n+1} p(T z, z) \preceq r p(T z, z)$.

Case 3. $p\left(T^{n+1} z, T z\right) \preceq r p(T z, z)$.

Case 4.

$$
\begin{aligned}
p\left(T^{n+1} z, T z\right) & \preceq r \frac{p\left(T^{n+1} z, z\right)+p\left(T z, T^{n} z\right)}{2} \preceq r\left(\frac{r^{n} p(T z, z)+r p(T z, z)}{2}\right) \\
& \preceq r^{2} p(T z, z) \preceq r p(T z, z) .
\end{aligned}
$$

Then, in all cases, we have $p\left(T^{n+1} z, T z\right) \preceq r p(T z, z)$. Therefore, (2.5) holds. Now, from (2.3) we have

$$
p\left(z, T^{n+1} z\right) \preceq r p\left(z, T^{n} z\right) \preceq \cdots \preceq r^{n} p(z, T z)
$$

for $n \geq 1$. Since $0 \leq r<1$, for every $c \gg \theta$, there exists $n_{0} \in N$ such that $p(T z, z) \ll c$ for all $n \geq n_{0}$. Hence,

$$
\begin{aligned}
p(z, T z) & \preceq p\left(z, T^{n+1} z\right)+p\left(T^{n+1} z, T z\right) \\
& \preceq r^{n} p(z, T z)+r p(z, T z) \ll c+r p(z, T z),
\end{aligned}
$$


which implies that $p(T z, z)=\theta$. Thus, $T z=z$. This is a contradiction.

In case (3), we note that for $x, y \in X$, either

$$
\psi(r) p(x, T x) \preceq p(x, y) \quad \text { or } \quad \psi(r) p\left(T x, T^{2} x\right) \preceq p(T x, y) .
$$

Indeed, if

$$
\psi(r) p(x, T x) \succ p(x, y) \quad \text { and } \quad \psi(r) p\left(T x, T^{2} x\right) \succ p(T x, y),
$$

then we have

$$
\begin{aligned}
p(x, T x) & \preceq p(x, y)+p(T x, y) \\
& \prec \psi(r)\left[p(x, T x)+p\left(T x, T^{2} x\right)\right] \\
& \prec \psi(r)[p(x, T x)+r p(x, T x)] \\
& =\left(\frac{1}{1+r}\right)(1+r) p(x, T x)=p(x, T x) .
\end{aligned}
$$

This is a contradiction. Now, since either

$$
\psi(r) p\left(x_{2 n}, T x_{2 n}\right) \preceq p\left(x_{2 n}, z\right) \quad \text { or } \quad \psi(r) p\left(x_{2 n+1}, T x_{2 n+1}\right) \preceq p\left(x_{2 n+1}, z\right)
$$

for all $n \in N$, by (2.1) it follows that either

$$
p\left(T x_{2 n}, T z\right) \preceq r U\left(x_{2 n}, z\right) \quad \text { or } \quad p\left(T x_{2 n+1}, T z\right) \preceq r U\left(x_{2 n+1}, z\right),
$$

where

$$
\begin{aligned}
& U\left(x_{2 n}, z\right) \in\left\{p\left(z, x_{2 n}\right), p\left(x_{2 n}, x_{2 n+1}\right), p(z, T z), \frac{p\left(x_{2 n}, T z\right)+p\left(z, x_{2 n+1}\right)}{2}\right\} \\
& U\left(x_{2 n+1}, z\right) \in\left\{p\left(z, x_{2 n+1}\right), p\left(x_{2 n+1}, x_{2 n+2}\right), p(z, T z), \frac{p\left(x_{2 n+1}, T z\right)+p\left(z, x_{2 n+2}\right)}{2}\right\} .
\end{aligned}
$$

Hence, we deduce that either

$$
p(T z, z) \preceq p\left(T x_{2 n}, z\right)+p\left(T x_{2 n}, T z\right)
$$

or

$$
p(T z, z) \preceq p\left(T x_{2 n+1}, z\right)+p\left(T x_{2 n+1}, T z\right) .
$$

Since $x_{n} \stackrel{\tau_{p}}{\rightarrow} z$ for every $c \gg \theta$, there exists $n_{0} \in N$ such that $p\left(x_{n}, z\right) \ll \frac{c}{2}$ and $p\left(x_{n}, x_{n+1}\right) \ll$ $\frac{c}{2}$ for all $n>n_{0}$. Now, by (2.6) we get the following cases:

Case 1. $p(T z, z) \preceq p\left(x_{2 n+1}, z\right)+r p\left(z, x_{2 n}\right) \ll c$ implies $p(T z, z)=\theta$.

Case 2. $p(T z, z) \preceq p\left(x_{2 n+1}, z\right)+r p\left(x_{2 n}, x_{2 n+1}\right) \ll c$ implies $p(T z, z)=\theta$.

Case 3. $p(T z, z) \preceq p\left(x_{2 n+1}, z\right)+r p(z, T z) \ll c+r p(z, T z)$ implies $p(T z, z)=\theta$. 
Case 4.

$$
\begin{aligned}
p(T z, z) & \preceq p\left(x_{2 n+1}, z\right)+r\left[\frac{p\left(x_{2 n}, T z\right)+p\left(z, x_{2 n+1}\right)}{2}\right] \\
& \preceq p\left(x_{2 n+1}, z\right)+r\left[\frac{p\left(x_{2 n}, z\right)+p(z, T z)+p\left(z, x_{2 n+1}\right)}{2}\right] \ll c+r p(z, T z),
\end{aligned}
$$

which implies that $p(T z, z)=\theta$. Then, in all cases, we have $p(T z, z)=\theta$. Similarly, by (2.7) we also have $p(T z, z)=\theta$. Thus, $T z=z$. This is a contradiction.

Therefore, in all cases, there exists $j \in N$ such that $T^{j} z=z$. Since $\left\{T^{n} z\right\}$ is a $\theta$-Cauchy sequence, we obtain $z=T z$. If not, that is, if $z \neq T z$, from $p\left(T^{n j} z, T^{n j+1} z\right)=p(z, T z)$ for all $n \in N$ it follows that $\left\{T^{n} z\right\}$ is not a $\theta$-Cauchy sequence. Hence, $z$ is a fixed point of $T$.

Finally, we prove the uniqueness of the fixed point. Suppose that there exist $z_{1}, z_{2} \in X$ with $z_{1} \neq z_{2}$ such that $T z_{1}=z_{1}$ and $T z_{2}=z_{2}$. We have two possible cases:

Case (a). If $z_{1}$ and $z_{2}$ are comparable, using (2.1) with $x=z_{1}$ and $y=z_{2}$, we get that

$$
p\left(z_{1}, z_{2}\right)=p\left(T z_{1}, T z_{2}\right) \preceq r U\left(z_{1}, z_{2}\right),
$$

where

$$
\begin{aligned}
U\left(z_{1}, z_{2}\right) & \in\left\{p\left(z_{1}, z_{2}\right), p\left(z_{1}, T z_{1}\right), p\left(z_{2}, T z_{2}\right), \frac{p\left(z_{1}, T z_{2}\right)+p\left(z_{2}, T z_{1}\right)}{2}\right\} \\
& =\left\{p\left(z_{1}, z_{2}\right), p\left(z_{1}, z_{1}\right), p\left(z_{2}, z_{2}\right), \frac{p\left(z_{1}, z_{2}\right)+p\left(z_{2}, z_{1}\right)}{2}\right\} \\
& =\left\{p\left(z_{1}, z_{2}\right), p\left(z_{1}, z_{1}\right), p\left(z_{2}, z_{2}\right)\right\} .
\end{aligned}
$$

Thus, we get the following cases:

Case 1. $p\left(z_{1}, z_{2}\right) \preceq r p\left(z_{1}, z_{2}\right)$ implies $p\left(z_{1}, z_{2}\right)=\theta$.

Case 2. $p\left(z_{1}, z_{2}\right) \preceq r p\left(z_{1}, z_{1}\right) \preceq r p\left(z_{1}, z_{2}\right)$ implies $p\left(z_{1}, z_{2}\right)=\theta$.

Case 3. $p\left(z_{1}, z_{2}\right) \preceq r p\left(z_{2}, z_{2}\right) \preceq r p\left(z_{1}, z_{2}\right)$ implies $p\left(z_{1}, z_{2}\right)=\theta$.

Thus, in all cases, we have $p\left(z_{1}, z_{2}\right)=\theta$, that is, $z_{1}=z_{2}$. This is a contradiction. Hence, $z_{1}=z_{2}$.

Case (b). If $z_{1}$ and $z_{2}$ are not comparable, then there exists $x \in X$ comparable with $z_{1}$ and $z_{2}$. First, we note that for each $x \in X$ comparable with $z_{1}$, we have that $T^{n} z_{1}$ and $T^{n} x$ are comparable and $\psi(r) p\left(T^{n-1} z_{1}, T^{n} z_{1}\right) \preceq p\left(T^{n-1} z_{1}, T^{n} z_{1}\right)=p\left(z_{1}, z_{1}\right) \preceq p\left(T^{n-1} z_{1}, T^{n-1} x\right)$. By (2.1) we obtain

$$
p\left(z_{1}, T^{n} x\right)=p\left(T^{n} z_{1}, T^{n} x\right) \preceq r U\left(T^{n-1} z_{1}, T^{n-1} x\right)
$$

where

$$
\begin{aligned}
U\left(T^{n-1} z_{1}, T^{n-1} x\right) \in & \left\{p\left(T^{n-1} z_{1}, T^{n-1} x\right), p\left(T^{n-1} z_{1}, T^{n} z_{1}\right), p\left(T^{n-1} x, T^{n} x\right),\right. \\
& \left.\frac{p\left(T^{n-1} x, T^{n} z_{1}\right)+p\left(T^{n-1} z_{1}, T^{n} x\right)}{2}\right\} \\
= & \left\{p\left(z_{1}, T^{n-1} x\right), p\left(z_{1}, z_{1}\right), p\left(T^{n-1} x, T^{n} x\right), \frac{p\left(z_{1}, T^{n-1} x\right)+p\left(z_{1}, T^{n} x\right)}{2}\right\},
\end{aligned}
$$


Thus, we get the following cases:

Case 1. $p\left(z_{1}, T^{n} x\right) \preceq r p\left(z_{1}, T^{n-1} x\right) \preceq r^{2} p\left(z_{1}, T^{n-2} x\right) \preceq \cdots \preceq r^{n} p\left(z_{1}, x\right)$.

Case 2. $p\left(z_{1}, T^{n} x\right) \preceq r p\left(z_{1}, z_{1}\right) \preceq r p\left(z_{1}, T^{n-1} x\right) \preceq \cdots \preceq r^{n} p\left(z_{1}, x\right)$.

Case 3. $p\left(z_{1}, T^{n} x\right) \preceq r p\left(T^{n-1} x, T^{n} x\right) \preceq r^{2} p\left(T^{n-2} x, T^{n-1} x\right) \preceq \cdots \preceq r^{n} p(x, T x)$.

Case 4. $p\left(z_{1}, T^{n} x\right) \preceq r\left[\frac{p\left(z_{1}, T^{n-1} x\right)+p\left(z_{1}, T^{n} x\right)}{2}\right]$ implies $p\left(z_{1}, T^{n} x\right) \preceq r p\left(z_{1}, T^{n-1} x\right) \preceq r^{n} p\left(z_{1}, x\right)$.

Thus, in all cases, we have

$$
p\left(z_{1}, T^{n} x\right) \preceq r^{n} p\left(z_{1}, x\right) \operatorname{or} p\left(z_{1}, T^{n} x\right) \preceq r^{n} p(x, T x) .
$$

Similarly, $p\left(z_{2}, T^{n} x\right) \preceq r^{n} p\left(z_{2}, x\right)$ or $p\left(z_{2}, T^{n} x\right) \preceq r^{n} p(x, T x)$. Let $\theta \ll c$ and choose a natural number $N_{3}$ such that $r^{n} p\left(z_{1}, x\right) \ll \frac{c}{2}$, or $r^{n} p(x, T x) \ll \frac{c}{2}$ and $r^{n} p\left(z_{2}, x\right) \ll \frac{c}{2}$ for all $n \geq N_{3}$. Thus,

$$
p\left(z_{1}, z_{2}\right) \preceq p\left(z_{1}, T^{n} x\right)+p\left(z_{2}, T^{n} x\right) \ll \frac{c}{2}+\frac{c}{2}=c,
$$

which is again a contradiction. Hence, $z_{1}=z_{2}$.

Now, in order to support the usability of Theorem 2.1, we present the following example.

Example 2.1 Let $E=C_{R}^{1}[0,1]$ with the norm $\|u\|=\|u\|_{\infty}+\left\|u^{\prime}\right\|_{\infty}$, and $X=P=\{u \in E$ : $u(t) \geq 0, t \in[0,1]\}$, which is a nonnormal solid cone. Define the mapping $p: X \times X \rightarrow P$ by

$$
p(x, y)= \begin{cases}x, & x=y \\ x+y & \text { otherwise }\end{cases}
$$

Then $(X, p)$ is a $\theta$-complete partial cone metric space. We can define a partial order on $X$ as follows:

$x \sqsubseteq y \quad$ if and only if $\quad x(t) \leq y(t) \quad$ for all $t \in[0,1]$.

Then $(X, p, \sqsubseteq)$ is a $\theta$-complete partially ordered partial cone metric space. For every fixed $r \in[0,1)$, define $T: X \rightarrow X$ by

$$
T x(t)= \begin{cases}0 & \text { if } t \in[0,1] \text { such that } x(t)=0, \\ \frac{2 n-1}{2} r t+\frac{2 n-1}{4 n} r x(t) & \text { if } t \in[0,1] \text { such that } 2 n-1 \leq x(t) \leq 2 n \text { for } n \in Z^{+}, \\ n r t+\frac{n}{2 n+1} r x(t) & \text { if } t \in[0,1] \text { such that } 2 n \leq x(t) \leq 2 n+1 \text { for } n \in Z^{+}\end{cases}
$$

Thus, for all $x \in X$, we consider the following three cases:

Case 1. If $t \in[0,1]$ such that $x(t)=0$, then $T x(t)=x(t)$.

Case 2. If $t \in[0,1]$ such that $2 n-1 \leq x(t) \leq 2 n$, then

$$
T x(t)=\frac{2 n-1}{2} r t+\frac{2 n-1}{4 n} r x(t) \leq \frac{2 n-1}{2} r+\frac{2 n-1}{2} r \leq r x(t) .
$$

Case 3. If $t \in[0,1]$ such that $2 n \leq x(t) \leq 2 n+1$, then

$$
T x(t)=n r t+\frac{n}{2 n+1} r x(t) \leq n r+n r \leq r x(t) .
$$


In all cases, for all $x \in X$ and $t \in[0,1]$, we have $T x(t) \leq r x(t)$. Hence, for all $x, y \in X, x \sqsubseteq y$, we have

$$
p(T x, T y) \preceq r p(x, y) \preceq r U(x, y),
$$

where $U(x, y) \in\left\{p(x, y), p(x, T x), p(y, T y), \frac{p(x, T y)+p(y, T x)}{2}\right\}$, which ensures that condition (2.1) is satisfied. Also, conditions (i)-(iii) of Theorem 2.1 are satisfied. Following the Theorem 2.1, we deduce that $T$ has a fixed point in $X$; indeed, $x=\theta$ is a fixed point of $T$.

Theorem 2.2 Let $(X, p$, ᄃ) be a partially ordered partial cone metric space over a solid cone $P$ of a normed vector space $(E,\|\cdot\|)$. Let $S, T: X \rightarrow X$ be such that $T$ is an $S$ nondecreasing mapping with respect to $\sqsubseteq, T X \subseteq S X$, and $S X$ is a $\theta$-complete subset of $X$. Define $\psi:[0,1) \rightarrow\left(\frac{1}{2}, 1\right]$ as in Theorem 2.1. Suppose that there exists $r \in[0,1)$ such that

$$
\psi(r) p(S x, T x) \preceq p(S x, S y) \quad \text { implies } \quad p(T x, T y) \preceq r U(S x, S y)
$$

for all comparable $S x, S y \in X$, where $U(S x, S y) \in\{p(S x, S y), p(S x, T x), p(S y, T y),(p(S x, T y)+$ $p(S y, T x)) / 2\}$. Suppose that the following conditions hold:

(i) there exists $x_{0} \in X$ such that $S x_{0} \sqsubseteq T x_{0}$;

(ii) for a nondecreasing sequence $x_{n} \stackrel{\tau_{p}}{\rightarrow} x$, we have $x_{n} \sqsubseteq x$ for all $n \in N$;

(iii) for two nondecreasing sequences $\left\{x_{n}\right\},\left\{y_{n}\right\} \subseteq X$ such that $x_{n} \sqsubseteq y_{n}, x_{n} \stackrel{\tau_{p}}{\rightarrow} x$, and $y_{n} \stackrel{\tau_{p}}{\rightarrow} y$ as $n \rightarrow \infty$, we have $x \sqsubseteq y$;

(iv) the set of points of coincidence of $S$ and $T$ is totally ordered, and $S, T$ are weakly compatible.

Then $S$ and $T$ have a unique common fixed point in $X$.

Proof By Lemma 1.1 there exists $Y \subseteq X$, such that $S Y=S X$ and $S: Y \rightarrow X$ is one-to-one. Define $f: S Y \rightarrow S X$ by $f S x=T x$ for all $S x \in S Y$.

Since $S$ is one-to-one on $Y, f$ is well defined. Note that, for all comparable $S x, S y \in S Y$,

$$
\psi(r) p(S x, f S x) \preceq p(S x, S y) \quad \text { implies } \quad p(f S x, f S y) \preceq r U(S x, S y)
$$

where $U(S x, S y) \in\left\{p(S x, S y), p(S x, f S x), p(S y, f S y), \frac{p(S x, f S y)+p(S y, f S x)}{2}\right\}$.

Since $T$ is $S$-nondecreasing, we have that $f$ is nondecreasing. In fact, $S x \sqsubseteq S y$ implies $T x \sqsubseteq T y$, and hence $f S x=T x \sqsubseteq T y=f S y$. Since $S Y$ is $\theta$-complete, by Theorem 2.1 we get that $f$ has a fixed point on $S Y$, say $S z$. Then $z=y$ is a coincidence point of $S$ and $T$, that is, $T z=f S z=S z$.

Now, we prove that $S$ and $T$ have a unique coincidence point. Suppose that $w$ is another coincidence point of $S$ and $T$ with $z \neq w$. Then

$$
\psi(r) p(S z, T z) \preceq p(S z, S w),
$$

and by (2.8) we have

$$
p(T z, T w) \preceq r U(S z, S w),
$$


where $U(S z, S w) \in\left\{p(S z, S w), p(S z, T z), p(S w, T w), \frac{p(S z, T w)+p(S w, T z)}{2}\right\}$. Since $S z=T z$ and $S w=$ $T w$, it follows from (2.9) that

$$
p(T z, T w) \preceq r U(T z, T w),
$$

where $U(T z, T w) \in\left\{p(T z, T w), p(T z, T z), p(T w, T w), \frac{p(T z, T w)+p(T w, T z)}{2}\right\}=\{p(T z, T w), p(T z, T z)$, $p(T w, T w)\}$, which is a contradiction. Hence, $z=w$. Let $v=S z=T z$. Since $S$ and $T$ are weakly compatible, we have $S v=S T z=T S z=T v$. Then $v$ is also a coincidence point of $S$ and $T$, Thus, $v=z$ by uniqueness. Therefore, $z$ is the unique common fixed point of $S$ and $T$.

\section{Coupled point theorems in partial cone metric spaces}

In this section, we will apply the results obtained in Section 2 to establish the corresponding Suzuki-type coupled fixed point theorems for generalized mappings in partially ordered partial cone metric spaces over a nonnormal cone.

For $\tilde{a}=(x, y), \tilde{b}=(u, v) \in X^{2}$, we introduce the mapping $\tilde{p}: X^{2} \times X^{2} \rightarrow P$ defined by $\tilde{p}(\tilde{a}, \tilde{b})=p(x, u)+p(y, v)$.

The following conclusion is valid, and for its proof, we refer to [30].

Lemma 3.1 If $(X, p)$ is a partial cone metric space over a solid cone $P$ of a normed vector space $(E,\|\cdot\|)$, then $\left(X^{2}, \tilde{p}\right)$ is also a $\theta$-complete partial cone metric space.

Proof It suffices to prove that, for $\tilde{a}=(x, y), \tilde{b}=(u, v), \tilde{c}=(z, w) \in X^{2}$,

$$
\tilde{p}(\tilde{a}, \tilde{b}) \preceq \tilde{p}(\tilde{a}, \tilde{c})+\tilde{p}(\tilde{b}, \tilde{c})-\tilde{p}(\tilde{c}, \tilde{c}) .
$$

In fact, for $\tilde{a}=(x, y), \tilde{b}=(u, v), \tilde{c}=(z, w) \in X^{2}$, we have

$$
\begin{aligned}
\tilde{p}(\tilde{a}, \tilde{b}) & =p(x, u)+p(y, v) \\
& \preceq p(x, z)+p(u, z)-p(z, z)+p(y, w)+p(v, w)-p(w, w) \\
& =p(x, z)+p(y, w)+p(u, z)+p(v, w)-[p(z, z)+p(w, w)] \\
& =\tilde{p}(\tilde{a}, \tilde{c})+\tilde{p}(\tilde{b}, \tilde{c})-\tilde{p}(\tilde{c}, \tilde{c}) .
\end{aligned}
$$

Suppose that the sequence $\left\{\tilde{x}_{n}\right\}=\left\{\left(x_{n}, y_{n}\right)\right\}$ is a $\theta$-Cauchy sequence in $\left(X^{2}, \tilde{p}\right)$. Then, for every $c \gg \theta$, there exists a positive integer $n_{0} \in N$ such that $\tilde{p}\left(\tilde{x}_{n}, \tilde{x}_{m}\right)=p\left(x_{n}, x_{m}\right)+$ $p\left(y_{n}, y_{m}\right) \ll c$ for all $n, m>n_{0}$. Then $p\left(x_{n}, x_{m}\right) \ll c$ and $p\left(y_{n}, y_{m}\right) \ll c$. Thus, $\left\{x_{n}\right\}$ and $\left\{y_{n}\right\}$ are $\theta$-Cauchy sequences in $(X, p)$. Since $(X, p)$ is $\theta$-complete, there exist $x, y \in X$ such that $x_{n} \stackrel{\tau_{p}}{\rightarrow} x, y_{n} \stackrel{\tau_{p}}{\rightarrow} y$, and $p(x, x)=\theta, p(y, y)=\theta$.

Thus, for every $c \gg \theta$, there exists $n_{1} \in N$ such that $p\left(x_{n}, x\right) \ll \frac{c}{2}$ and $p\left(y_{n}, y\right) \ll \frac{c}{2}$ for all $n>n_{1}$. Then $\tilde{p}\left(\left(x_{n}, y_{n}\right),(x, y)\right)=p\left(x_{n}, x\right)+p\left(y_{n}, y\right) \ll \frac{c}{2}+\frac{c}{2}=c$, and $\tilde{p}((x, y),(x, y))=\theta$. Thus, $\left\{\left(x_{n}, y_{n}\right)\right\} \stackrel{\tau_{\tilde{p}}}{\rightarrow}(x, y)$.

Therefore, $\left(X^{2}, \tilde{p}\right)$ is a $\theta$-complete partial cone metric space.

Theorem 3.1 Let $(X, p$, ᄃ) be a $\theta$-complete partially ordered partial cone metric space over a solid cone $P$ of a normed vector space $(E,\|\cdot\|)$. Let $A: X \times X \rightarrow X$ be a mapping 
satisfying the mixed monotone property on $X$ with respect to $\sqsubseteq$. Define $\psi:[0,1) \rightarrow\left(\frac{1}{2}, 1\right]$ as in Theorem 2.1. Assume that there exists $r \in[0,1)$ such that

$$
\begin{aligned}
& \psi(r)[p(x, A(x, y))+p(y, A(y, x))] \preceq p(x, u)+p(y, v) \quad \text { implies } \\
& p(A(x, y), A(u, v))+p(A(y, x), A(v, u)) \preceq r U((x, y),(u, v))
\end{aligned}
$$

for all $x, y \in X$ such that $x \sqsubseteq u$ and $v \sqsubseteq y$, where

$$
\begin{aligned}
U((x, y),(u, v)) \in & \{p(x, u)+p(y, v), p(x, A(x, y))+p(y, A(y, x)), \\
& p(u, A(u, v))+p(v, A(v, u)), \\
& \left.\frac{p(x, A(u, v))+p(y, A(v, u))+p(u, A(x, y))+p(v, A(v, u))}{2}\right\} .
\end{aligned}
$$

Suppose that the following conditions hold:

(i) there exists $x_{0}, y_{0} \in X$ such that $x_{0} \sqsubseteq A\left(x_{0}, y_{0}\right)$ and $A\left(y_{0}, x_{0}\right) \sqsubseteq y_{0}$;

(ii) for a nondecreasing sequence $x_{n} \stackrel{\tau_{p}}{\rightarrow} x$, we have $x_{n} \sqsubseteq x$ for all $n \in N$;

(iii) for a nonincreasing sequence $y_{n} \stackrel{\tau_{p}}{\rightarrow} x$, we have $y \sqsubseteq y_{n}$ for all $n \in N$;

(iv) for two nondecreasing sequences $\left\{x_{n}\right\},\left\{u_{n}\right\} \subseteq X$ such that $x_{n} \sqsubseteq u_{n}$ for all $n \in N$, $x_{n} \stackrel{\tau_{p}}{\rightarrow} x$, and $u_{n} \stackrel{\tau_{p}}{\rightarrow} u$ as $n \rightarrow \infty$, we have $x \sqsubseteq u$;

(v) for two nonincreasing sequences $\left\{y_{n}\right\},\left\{v_{n}\right\} \subseteq X$ such that $v_{n} \sqsubseteq y_{n}$ for all $n \in N$, $v_{n} \stackrel{\tau_{p}}{\rightarrow} v$, and $y_{n} \stackrel{\tau_{p}}{\rightarrow}$ y as $n \rightarrow \infty$, we have $v \sqsubseteq y$.

Then $A$ has a coupled fixed point in $X$, that is, there exist $z, w \in X$ such that $A(z, w)=z$ and $A(w, z)=w$.

Proof Let $\tilde{X}=X \times X$. For $\tilde{a}=(x, y), \tilde{b}=(u, v) \in \tilde{X}$, we introduce the order $\prec$ as

$$
\tilde{a} \prec \tilde{b} \quad \text { if and only if } \quad x \sqsubseteq u, \quad v \sqsubseteq y .
$$

It follows from Lemma 3.1 that $(\tilde{X}, \tilde{p}, \prec)$ is also a $\theta$-complete partially ordered partial cone metric space, where

$$
\tilde{p}(\tilde{a}, \tilde{b})=p(x, u)+p(y, v) .
$$

The mapping $T: \tilde{X} \rightarrow \tilde{X}$ is given by $T \tilde{a}=(A(x, y), A(y, x))$ for all $\tilde{a}=(x, y) \in \tilde{X}$. Then a coupled point of $A$ is a fixed point of $T$ and vice versa.

If $\tilde{a} \prec \tilde{b}$, then $x \sqsubseteq u$ and $v \sqsubseteq y$. Noting the mixed monotone property of $A$, we see that if $A(x, y) \sqsubseteq A(u, v)$ and $A(v, u) \sqsubseteq A(y, x)$, then $T \tilde{a} \prec T \tilde{b}$. Thus, $T$ is a nondecreasing mapping with respect to the order $\prec$ on $\tilde{X}$.

On the other hand, for all $\tilde{a}=(x, y), \tilde{b}=(u, v) \in \tilde{X}$ with $\tilde{a} \prec \tilde{b}$, if $\psi(r) \tilde{p}(\tilde{a}, T \tilde{a})=$ $\psi(r)[p(x, A(x, y))+p(y, A(y, x))] \preceq p(x, u)+p(y, v)=\tilde{p}(\tilde{a}, \tilde{b})$, then we have

$$
\tilde{p}(T \tilde{a}, T \tilde{b})=p(A(x, y), A(u, v))+p(A(y, x), A(v, u)) \preceq r U((x, y),(u, v)),
$$


where

$$
\begin{aligned}
U(\tilde{a}, \tilde{b})= & U((x, y),(u, v)) \\
\in & \{p(x, u)+p(y, v), p(x, A(x, y))+p(y, A(y, x)), p(u, A(u, v))+p(v, A(v, u)), \\
& \left.\frac{p(x, A(u, v))+p(y, A(v, u))+p(u, A(x, y))+p(v, A(v, u))}{2}\right\} \\
= & \left\{\tilde{p}(\tilde{a}, \tilde{b}), \tilde{p}(\tilde{a}, T \tilde{a}), \tilde{p}(\tilde{b}, T \tilde{b}), \frac{\tilde{p}(\tilde{a}, T \tilde{b})+\tilde{p}(T \tilde{a}, \tilde{b})}{2}\right\} .
\end{aligned}
$$

Also, there exists an $\tilde{x}_{0}=\left(x_{0}, y_{0}\right) \in \tilde{X}$ such that $\tilde{x}_{0} \prec T \tilde{x}_{0}=\left(A\left(x_{0}, y_{0}\right), A\left(y_{0}, x_{0}\right)\right)$.

If a nondecreasing monotone sequence $\left\{\tilde{x}_{n}\right\}=\left\{\left(x_{n}, y_{n}\right)\right\}$ in $\tilde{X} \tau_{p}$-converges to $\tilde{x}=(x, y)$, then $\tilde{x}_{n}=\left(x_{n}, y_{n}\right) \prec\left(x_{n+1}, y_{n+1}\right)=\tilde{x}_{n+1}$, that is, $x_{n} \sqsubseteq x_{n+1}$ and $y_{n+1} \sqsubseteq y_{n}$. Thus, $\left\{x_{n}\right\}$ is a nondecreasing sequence $\tau_{p}$-converging to $x$, and $\left\{y_{n}\right\}$ is a nonincreasing sequence $\tau_{p}$-converging to $y$. Thus, $x_{n} \sqsubseteq x$ and $y \sqsubseteq y_{n}$ for all $n \in N$. This implies $\tilde{x}_{n} \prec \tilde{x}$.

If two nondecreasing sequence $\left\{\tilde{x}_{n}\right\}=\left\{\left(x_{n}, y_{n}\right)\right\},\left\{\tilde{y}_{n}\right\}=\left\{\left(u_{n}, v_{n}\right)\right\}$ are such that $\tilde{x}_{n} \prec \tilde{y}_{n}$ for all $n \in N, \tilde{x}_{n} \stackrel{\tau_{\tilde{p}}}{\rightarrow}(x, y)$, and $\tilde{y}_{n} \stackrel{\tau_{\tilde{p}}}{\rightarrow}(u, v)$ as $n \rightarrow \infty$, then $x_{n} \sqsubseteq u_{n}, v_{n} \sqsubseteq y_{n}, x_{n} \sqsubseteq x_{n+1}, y_{n+1} \sqsubseteq y_{n}$, and $u_{n} \sqsubseteq u_{n+1}, v_{n+1} \sqsubseteq v_{n}$. Thus, $\left\{x_{n}\right\},\left\{u_{n}\right\} \subseteq X$ are two nondecreasing sequences, $x_{n} \sqsubseteq u_{n}$ for all $n \in N, x_{n} \stackrel{\tau_{p}}{\rightarrow} x$, and $u_{n} \stackrel{\tau_{p}}{\rightarrow} u$ as $n \rightarrow \infty$, and by condition (iv) we have $x \sqsubseteq u$. Similarly, by condition (v) we have $v \sqsubseteq y$. Thus, $(x, y) \prec(u, v)$.

Therefore, all hypotheses of Theorem 2.1 are satisfied. Following Theorem 2.1, we deduce that $A$ has a coupled point, that is, there exist $z, w \in \tilde{X}$ such that $A(z, w)=z$ and $A(w, z)=w$.

Now, we present the following example.

Example 3.1 Let $X=P=\left\{\left(x_{1}, x_{2}\right): x_{1}, x_{2} \in R^{+}\right\} \subseteq R^{2}$, and $E=R^{2}$ with the norm $\|x\|=$ $\sqrt{x_{1}^{2}+x_{2}^{2}}$. Define the mapping $p: X \times X \rightarrow P$ by

$$
p(x, y)=\left(x_{1} \vee y_{1}, x_{2} \vee y_{2}\right)
$$

for all $x=\left(x_{1}, x_{2}\right), y=\left(y_{1}, y_{2}\right) \in X$, where $a \vee b=\max \{a, b\}, a, b \in R^{+}$. Then $(X, p)$ is a $\theta$ complete partial cone metric space. Define the partial order on $X$ by

$$
x \sqsubseteq y \quad \text { if and only if } \quad x_{1} \leq x_{2}, \quad y_{1} \leq y_{2} .
$$

Then $(X, p, \sqsubseteq)$ is a $\theta$-complete partially ordered partial cone metric space. In fact, " $\sqsubseteq$ " is equal to " $\preceq$ ". For any fixed $r \in[0,1)$, define $A: X \times X \rightarrow X$ by

$$
A(x, y)=A\left(\left(x_{1}, x_{2}\right),\left(y_{1}, y_{2}\right)\right)=\left(\frac{r x_{1}}{1+y_{1}}, \frac{r x_{2}}{e^{y_{2}}}\right)
$$

for all $x, y \in X$. It is clear that $A$ satisfies the mixed monotone property on $X$ with respect to $\sqsubseteq$. Define $\psi:[0,1) \rightarrow\left(\frac{1}{2}, 1\right]$ as in Theorem 3.1. 
Now, for all $x, y, u, v \in X, x \sqsubseteq u, v \sqsubseteq y$, we have

$$
\begin{aligned}
\psi(r)[p(x, A(x, y))+p(y, A(y, x))] & \preceq p(x, A(x, y))+p(y, A(y, x)), \\
& =\left(x_{1} \vee \frac{r x_{1}}{1+y_{1}}, x_{2} \vee \frac{r x_{2}}{e^{y_{2}}}\right)+\left(y_{1} \vee \frac{r y_{1}}{1+x_{1}}, y_{2} \vee \frac{r y_{2}}{e^{x_{2}}}\right) \\
& =\left(x_{1}+y_{1}, x_{2}+y_{2}\right) \\
& \preceq\left(u_{1}+y_{1}, u_{2}+y_{2}\right)=p(x, u)+p(y, v)
\end{aligned}
$$

and, on the other hand,

$$
\begin{aligned}
p & (A(x, y), A(u, v))+p(A(y, x), A(v, u)) \\
& =\left(\frac{r x_{1}}{1+y_{1}} \vee \frac{r u_{1}}{1+v_{1}}, \frac{r x_{2}}{e^{y_{2}}} \vee \frac{r u_{2}}{e^{\nu_{2}}}\right)+\left(\frac{r y_{1}}{1+x_{1}} \vee \frac{r v_{1}}{1+u_{1}}, \frac{r y_{2}}{e^{x^{2}}} \vee \frac{r v_{2}}{e^{u_{2}}}\right) \\
& =\left(\frac{r u_{1}}{1+v_{1}}+\frac{r y_{1}}{1+x_{1}}, \frac{r u_{2}}{e^{v_{2}}}+\frac{r y_{2}}{e^{x^{2}}}\right) \\
& \leq\left(r u_{1}+r y_{1}, r u_{2}+r y_{2}\right)=r[p(x, u)+p(y, v)] \\
& \leq r U((x, y),(u, v)),
\end{aligned}
$$

where $U((x, y),(u, v))$ is as in Theorem 3.1.

Also, conditions (i)-(v) of Theorem 3.1 are satisfied. From Theorem 2.1 we obtain that $A$ has a fixed point in $X$; indeed, $x=(0,0)$ is a fixed point of $A$.

\section{Competing interests}

The authors declare that they have no competing interests.

\section{Authors' contributions}

All authors contributed equally and significantly in writing this article. All authors read and approved the final manuscript.

\section{Author details}

${ }^{1}$ Department of Mathematics, Nanchang University, Nanchang, 330031, P.R. China. ${ }^{2}$ Department of Mathematics, Fengcheng No. 2 Senior School, Yichun, Jiangxi 331100, P.R. China.

\section{Acknowledgements}

The authors thank the editor and the referees for their valuable comments and suggestions. The research has been supported by the National Natural Science Foundation of China $(11071108,11361042,11326099,11461045)$ and the Provincial Natural Science Foundation of Jiangxi, China (2010GZS0147, 20114BAB201007, 20142BAB211004) and supported partly by the Provincial Graduate Innovation Foundation of Jiangxi, China (YC2012-B004).

Received: 14 July 2015 Accepted: 31 March 2016 Published online: 06 April 2016

\section{References}

1. Matthews, SG: Partial metric topology. In: Proc. 8th Summer Conference on General Topology and Applications. Ann. New York Acad. Sci., vol. 728, pp. 183-197 (1994)

2. Oltra, S, Valero, O: Banach's fixed point theorem for partial metric spaces. Rend. Ist. Mat. Univ. Trieste 36, 17-26 (2004)

3. Altun, I, Sola, F, Simsek, H: Generalized contractions on partial metric spaces. Topol. Appl. 157, 2778-2785 (2010)

4. Agarwal, RP, Alghamdi, MA, Shahard, N: Fixed point theory for cyclic generalized contractions in partial metric spaces. Fixed Point Theory Appl. 2012, 40 (2012)

5. Huang, LG, Zhang, X: Cone metric spaces and fixed point theorems of contractive mappings. J. Math. Anal. Appl. 332, $1468-1476$ (2007)

6. Rezapour, SH, Hamlbarani, R: Some notes on the paper "Cone metric spaces and fixed point theorems of contractive mappings". J. Math. Anal. Appl. 345, 719-724 (2008)

7. Arshad, M, Azam, A, Beg, I: Common fixed points of two maps in cone metric spaces. Rend. Circ. Mat. Palermo 57 , 433-441 (2008)

8. Bari, CD, Vetro, P: $\varphi$-Pairs and common fixed points in cone metric spaces. Rend. Circ. Mat. Palermo 57, 279-285 (2008)

9. Khamsi, M: Remarks on cone metric spaces and fixed point theorems of contractive mappings. Fixed Point Theory Appl. 2010, Article ID 315398 (2010) 
10. Vetro, P: Common fixed points in cone metric spaces. Rend. Circ. Mat. Palermo 56, 464-468 (2007)

11. Arshad, M, Azam, A, Vetro, P: Some common fixed point results in cone metric spaces. Fixed Point Theory Appl. 2009 Article ID 493965 (2009)

12. Turkoglu, M, Abuloha, M: Cone metric spaces and fixed point theorems in diametrically contractive mappings. Acta Math. Appl. Sinica (Engl. Ser.) 26, 489-496 (2010)

13. Altun, I, Damjanović, B, Djiorić, D: Fixed point and common fixed point theorems on ordered cone metric spaces. Appl. Math. Lett. 23, 310-316 (2010)

14. Hussaina, N, Shah, MH: KKM mappings in cone b-metric spaces. Comput. Math. Appl. 62, 1677-1684 (2011)

15. Roshan, JR, Parvaneh, V, Altun, I: Some coincidence point results in ordered b-metric spaces and applications in a system of integral equations. Appl. Math. Comput. 226, 725-737 (2014)

16. Sonmez, A: Fixed point theorems in partial cone metric spaces (2011). arXiv:1 101.2741v1 [math.GN]

17. Sonmez, A: On partial cone metric spaces (2012). arXiv:1207.6766v1 [math.GN]

18. Malhotra, SK, Shukla, S, Sen, R: Some fixed point results in $\theta$-complete partial cone metric spaces. J. Adv. Math. Stud. 6(2), 97-108 (2013)

19. Jiang, SJ, Li, ZL: Extensions of Banach contraction principle to partial cone metric spaces over a non-normal solid cone. Fixed Point Theory Appl. 2013, 250 (2013)

20. Bhaskar, TG, Lakshmikantham, V: Fixed point theory in partially ordered metric spaces and applications. Nonlinear Anal. 65, 1379-1393 (2006)

21. Haghi, RH, Rezapour, S, Shahzad, N: Some fixed point generalized are not real generalizations. Nonlinear Anal. 74, 1799-1803 (2011)

22. Paesano, D, Vetro, P: Suzuki's type characterizations of completeness for partial metric spaces and fixed points for partially ordered metric spaces. Topol. Appl. 159, 911-920 (2012)

23. Suzuki, T: A generalized Banach contraction principle that characterizes metric completeness of completeness. Proc. Am. Math. Soc. 136, 1861-1869 (2008)

24. Abbas, M, Ali, B, Vetro, C: A Suzuki type fixed point theorem for a generalized multivalued mapping on partial Hausdorff metric spaces. Topol. Appl. 160, 553-563 (2013)

25. Salimi, P, Vetro, P: A result of Suzuki type in partial G-metric spaces. Acta Math. Sci. 34B(2), 274-284 (2014)

26. Semwal, P, Dimri, RC: A Suzuki type coupled fixed point theorem for generalized multivalued mapping. Abstr. Appl. Anal. 2014, Article ID 820482 (2014)

27. Zhu, CX: Research on some problems for nonlinear operators. Nonlinear Anal. 71, 4568-4571 (2009)

28. Zhu, CX: Several nonlinear operator problems in the Menger PN space. Nonlinear Anal. 65, 1281-1284 (2006)

29. Zhu, CX, Xu, ZB: Inequalities and solution of an operator equation. Appl. Math. Lett. 21, 607-611 (2008)

30. Kadelburg, Z, Nashine, HK, Radenović, S: Coupled fixed points in 0-complete ordered partial metric spaces. J. Adv. Math. Stud. 6(1), 159-172 (2013)

\section{Submit your manuscript to a SpringerOpen ${ }^{\circ}$ journal and benefit from:}

- Convenient online submission

Rigorous peer review

- Immediate publication on acceptance

- Open access: articles freely available online

- High visibility within the field

- Retaining the copyright to your article 